\title{
Deformidade angular adquirida no carpo de muares: diagnóstico, incidência e tratamento
}

\author{
Acquired carpal angular limb deformities in mules: diagnosis, incidence and treatment
}

\author{
Bruno Zambelli Loiacono ${ }^{I^{*}}$ José Ramón Martinez Aranzales $^{\mathrm{I}, \mathrm{II}}$ Rafael de Resende Faleiros ${ }^{\mathrm{I}}$ \\ Geraldo Eleno Silveira Alves ${ }^{\mathrm{I}}$
}

\section{RESUMO}

\begin{abstract}
O objetivo deste trabalho foi diagnosticar, tratar $e$ monitorar a ocorrência de DA adquirida no carpo de muares. O tratamento instituído nos animais portadores de DA foi determinado pelo desvio angular mensurado em imagens radiológicas. Membros com desvio $\leq 3^{\circ}$ foram submetidos ao casqueamento (TC); desvios $>3^{\circ} e \leq 7^{\circ}$ foram tratados com transecção e elevação de periósteo e casqueamento (TEP), desvio $>7^{\circ}$ foram submetidos às mesmas medidas adotadas no grupo TEP, associadas ao uso de grampo trans-fisário (GTF). Os desvios foram monitorados através de radiografias obtidas 45 dias antes (TO), no início do tratamento (T1) e com 30 (T2) e 60 (T3) dias após a sua instituição. A incidência de animais acometidos no plantel estudado foi de $37,1 \%$, sendo a demanda por tratamento cirúrgico maior do que a relatada em equinos. $O$ fator determinante para a incidência de DA neste plantel foi o programa de treinamento instituído. Entre TO e T1, não houve variação da DA. No grupo TEP, a redução a partir de T2 foi semelhante à relatada nos equinos, porém a melhora ocorreu em menos tempo e em muares com idade de 7,9 meses. Não foi obtida redução satisfatória do desvio no grupo GTF, provavelmente, devido à média de idade elevada desse grupo. Conclui-se que os exercícios acarretaram na incidência de DA nos muares. O tratamento instituído no grupo TEP foi capaz de reduzir a DA, apesar da idade dos muares.
\end{abstract}

Palavras-chave: carpo, muar, valgo, varo.

\section{ABSTRACT}

The objective of this study was to diagnose, treat and monitor the occurrence of acquired carpal angular limb deformities (ALD) in a herd of mules. The treatment was determined by the degrees of angulation (DA) measured during the radiological examination. Members with $D A \leq 3^{\circ}$ were subjected to hoof trimming (TC); $D A>3^{\circ}$ and $\leq 7^{\circ}$ were treated with hemicircunfential transection and elevation of the periosteum and hoof trimming (TEP); DA $>7^{\circ}$ were subjected to the same measures adopted in the group TEP associated with transphyseal staple (GTF). The DA was monitored by radiography obtained 45 days before treatment (TO), at the start of treatment (T1) and 30 (T2) and 60 (T3) after its establishment. The incidence of affected mules was $37.1 \%$, in which the demand for surgical treatment was higher than that reported in horses. The occurrence of DA in this herd was due to the training program established. Between TO and T1 there was no variation of the DA. In the TEP group, the reduction of $D A$ in $T 2$ was similar to those reported in horses, but the improvement occurred in less time and in mules at the age of 7.9 months. The DA at T3 in the GTF was not satisfactory, probably due to the high average age of this group. It was concluded that the exercises resulted in the incidence of DA in mules. The adopted treatment in the TEP group was able to reduce the DA, despite the age of mules.

Key words: carpal, mules, valgus, varus.

\section{INTRODUÇÃo}

A deformidade angular (DA) adquirida é consequência de distúrbios do processo de ossificação endocondral das placas epifisárias decorrentes de dieta desbalanceada e trauma biomecânico (WITTE \& HUNT, 2009). O excesso de energia e a deficiência de macro e micro minerais são os principais fatores

IPrograma de Pós-graduação em Ciência Animal, Departamento de Clínica e Cirurgia Veterinárias, Universidade Federal de Minas Gerais (UFMG). Av. Antônio Carlos, 6627, 30123-970, Belo Horizonte, MG, Brasil. E-mail: bzambelli@gmail.com. *Autor para correspondência.

IIEscuela de Medicina Veterinaria, Universidad de Antioquia, Medellin, Antioquia, Colombia. 
nutricionais envolvidos (BERTONE, 2002). Isso resulta em incidências de DA variáveis nos equinos.

Potros da raça Puro Sangue Inglês apresentaram uma ocorrência de 62,1\% (O'DONOHUE et al., 1992), entretanto, ALDRED (1998) estimou que $80 \%$ dos potros são acometidos com algum grau de DA. Dentre as incidências relatadas, $85,7 \%$ a $87,1 \%$ dos potros têm sido acometidos por carpus valgus e $14,3 \%$ a $22,9 \%$ por carpus varus (PHARR \& FRETZ, 1981; BERTONE et al., 1985). CASTON et al. (2007) avaliaram 63 potros, dentre os quais $31,7 \%$ apresentaram carpus valgus bilateral, 50,7\% carpus valgus unilateral e $4,7 \%$ carpus varus unilateral.

O tipo de tratamento para DA do carpo é recomendado de acordo com o desvio angular da articulação e a idade do potro (AUER \& VON RECHENBERG, 2006). Recomenda-se a restrição de movimento e o casqueamento nos casos de DA discretas (AUER \& VON RECHENBERG, 2006), a transecção e elevação de periósteo em animais jovens e com DA moderada (AUER \& MARTENS, 1982) e colocação de pontes trans-fisárias em potros de idade mais avançada e/ou DA intensas. Os grampos transfisários, apesar de pouco utilizados atualmente, foram aplicados com sucesso para a correção de DA do carpo de potros (CASTON et al., 2007).

Atualmente, o rebanho de muares tem apresentado expansão significativa, além de aumento do valor comercial dos animais. Apesar desse crescimento, são escassas as informações referentes à DA adquirida na espécie. Portanto, o objetivo do presente estudo foi avaliar os fatores predisponentes de DA, a sua incidência e a resposta a diversos protocolos de tratamento em um rebanho de muares.

\section{MATERIAL E MÉTODOS}

Foram avaliados 35 muares com idade entre cinco e 20 meses, procedentes de cruzamentos de jumentos da raça Pega com éguas da raça Mangalarga Marchador, Appaloosa e sem raça definida, nascidos durante as estações reprodutivas 2007-2008 (oito animais) e 2008-2009 (27 animais). A geração mais velha foi identificada como G1 e a geração mais nova como G2. Através de inspeção clínica, foram selecionados os muares portadores de DA no carpo, sendo que quatro pertenciam a G1 e nove a G2. Todos foram medicados contra endo e ectoparasitas e alocados em piquetes de $3.000 \mathrm{~m}^{2}$.

Para avaliar a possibilidade de influência nutricional e de manejo na incidência de DA no plantel, foi investigada a presença de fatores predisponentes de DA. Avaliou-se a idade de desmame, área de criação, oferta de concentrados, disponibilidade de sal mineral, além de particularidades do treinamento dos muares no criatório. O tratamento de cada membro acometido por DA foi instituído de acordo com a intensidade do desvio angular, conforme recomendado por AUER \& VON RECHENBERG (2006). Para isso, foi obtida radiografia digital em projeção dorsopalmar do carpo dos treze muares através da utilização de emissor de raios-X Dong-A Ray e scanner Scan-X. Para determinar o desvio angular da articulação cárpica, foi mensurado com auxílio de ferramentas do software TigerView CD Viewer ${ }^{\circledR} \mathrm{o}$ ângulo formado entre os eixos axiais do rádio e metacarpiano III.

A conduta de tratamento instituída variou segundo o desvio angular, em cada membro, adotandose o seguinte critério: $\leq 3^{\circ}=$ casqueamento $(\mathrm{TC}) ;>3^{\circ}$ e $\leq 7^{\circ}=$ transecção e elevação de periósteo e casqueamento $(\mathrm{TEP}) ;>7^{\circ}=$ tratamento instituído em TEP associado à colocação de grampo de aço inox trans-epifisário (GTF). A partir desses critérios, diagnosticou-se DA no carpo em 13 animais. Entre os 26 membros avaliados, 16 foram alocados no grupo TC, seis no TEP e quatro no GTF, sendo permitido que o mesmo animal tivesse seus membros alocados em grupos diferentes. O casqueamento foi realizado em todos os membros, de acordo com as recomendações de GREET \& CURTIS (2003) e repetido após 30 e 60 dias após, sendo o primeiro realizado no dia anterior à cirurgia. No mesmo dia, foi avaliado o risco cirúrgico dos animais através de exame clínico e exames laboratoriais de rotina. Além disso, instituiu-se jejum alimentar e hídrico de 12 e 2 horas, respectivamente.

Como pré-medicação anestésica, utilizou-se xilazina $\left(0,8 \mathrm{mg} \mathrm{kg}^{-1} \mathrm{IV}^{-1}\right)$ e diazepam $\left(0,08 \mathrm{mg} \mathrm{kg}^{-1} \mathrm{IV}^{-1}\right)$ após cinco minutos. A indução anestésica foi realizada com cetamina $\left(2 \mathrm{mg} \mathrm{kg}^{-1} \mathrm{IV}^{-1}\right)$ e a manutenção anestésica através de infusão contínua $\left(2 \mathrm{a} 4 \mathrm{~mL} \mathrm{~kg}^{-1} \mathrm{~h}^{-1}\right)$ da solução a base de éter gliceril guaiacol (5\%), xilazina ( $0,05 \%)$ e cetamina (1\%), diluídos em solução glicosada a $5 \%$. Durante o início da manutenção anestésica, foi realizada tricotomia, antissepsia e colocação da bandagem de Smarch, no membro a ser operado.

Após a localização da placa epifisária distal do rádio, o procedimento de transecção e elevação do periósteo foi realizado conforme descrito por AUER \& VON RECHENBERG (2006). A técnica adotada para a colocação do grampo trans-fisário foi a descrita por CASTON et al. (2007). Após 60 dias, os grampos foram retirados seguindo as recomendações dos mesmos autores. Na técnica de elevação e transecção de periósteo e na colocação e remoção dos grampos transfisários, a sutura do tecido subcutâneo foi realizada em padrão festonado e, na pele, foi feita sutura intra- 
dérmica. $\mathrm{O}$ fio utilizado em todas as suturas foi o categute cromado $2-0$.

A ferida cirúrgica foi protegida imediatamente após o término da sutura por gaze enrolada e umedecida com rifamicina tópica $1 \%$, algodão hidrófobo, atadura de crepon e bandagem elástica autoaderente. Esse curativo permaneceu no local por até dez dias. Após a recuperação anestésica, foi iniciado tratamento com sulfametoxazol $\left(25 \mathrm{mg} \mathrm{kg}^{-1} \mathrm{VO}^{-1} \mathrm{SID}^{-1}\right)$ por cinco dias e com fenilbutazona $\left(4,4 \mathrm{mg} \mathrm{kg}^{-1} \mathrm{VO}^{-1}\right.$ $\mathrm{SID}^{-1}$ ) por três dias.

Durante o período de monitoramento, os muares permaneceram durante os primeiros dez dias em baias e retornaram em seguida para os piquetes de $3.000 \mathrm{~m}^{2}$. A resposta dos muares ao tratamento foi avaliada através de exames radiológicos realizados 45 dias antes (T0), no dia anterior (T1), e com 30 (T2) e 60 (T3) dias após a instituição dos tratamentos.

Neste experimento, o membro foi considerado como unidade experimental. A angulação da DA da articulação do carpo foi estudada através de delineamento inteiramente casualisado com covariância em arranjo em parcelas subdividas, sendo os tratamento as parcelas e os tempos as subparcelas. As médias foram comparadas pelo teste t-Student ou Tukey. Para os dados não paramétricos, os tratamentos foram comparados em cada tempo pelo teste de Kruskal-Wallis e os tempos foram comparados em cada tratamento pelo teste de Friedman.

\section{RESULTADOS E DISCUSSÃO}

Os muares, quando recém-nascidos, foram mantidos com as éguas sob manejo de pastejo em Brachiaria humidicola e capim de Andropogon gayanus e suplementados com sal mineral na época de chuvas e sal mineral proteinado na época seca. Esta dieta é pobre em nutrientes energéticos, o que exclui o excesso de energia como fator predisponente à ocorrência de DA.

Todos os muares com quatro meses de idade foram submetidos a exercícios físicos forçados em redondel de $16 \mathrm{~m}$ de diâmetro com piso de areia durante uma hora/dia, totalizando 18 sessões em três semanas. O propósito do treinamento era desenvolvimento de massa muscular. Os animais da G1 foram submetidos duas vezes a esse protocolo de exercício, sendo o primeiro em 2008 e o segundo em 2009, este simultaneamente com a G2. A ocorrência de DA foi observada em animais das duas gerações apenas durante o treinamento simultâneo de G1 e G2. Ao serem observadas evidências da afecção, o treinamento foi suspenso.
Apesar do trauma biomecânico não causar DA isoladamente (MCILWRAITH, 2004), neste trabalho, ficou claro que a instituição do treinamento nos muares jovens acarretou em DA adquirida. Esse achado corrobora as observações feitas por LEPEULE et al. (2009), que relataram predisposição de DA 2,8 vezes maior em potros equinos submetidos a protocolos de treinamento. Além disso, é possível que os muares avaliados apresentassem deficiência mineral, devido à ingestão de oxalato presente nas pastagens de Brachiaria humidicola (PUOLI FILHO et al., 1999). Tal ingestão poderia acarretar deficiência de cálcio, o que predispõe a ocorrência de DA (ALDRED, 1998).

Dos 35 muares examinados clinicamente, 13 $(37,1 \%)$ apresentaram DA bilateral na articulação cárpica, sendo quatro pertencentes a G1 e nove a G2. A incidência de DA foi de $50 \%$ na G1 e de $33,3 \%$ na G2, enquanto a média de idades no dia da instituição dos tratamentos foi de 20 e 7,9 meses, respectivamente. Entre os animais portadores de DA, 66,9\% apresentaram carpus valgus e $23,1 \%$ carpus varus, sendo que todos os muares apresentavam o mesmo tipo de DA em ambos os membros. O número de animais portadores de DA, quantidade de membros acometidos e o tipo de DA estão especificados para cada grupo na tabela 1. É importante enfatizar que nesse experimento foram avaliados 13 muares portadores de DA. Entretanto, na tabela 1, o número total apontado é de 16 animais. Essa diferença é decorrente do fato de um animal da G1 e dois da G2, todos portadores de carpus valgus, terem um membro alocado no grupo TC e o outro no grupo TEP.

Estudos epidemiológicos de incidência de DA em equinos mostraram índices elevados entre 62,1 e 80\% (ALDRED, 1998; O'DONOHUE et al., 1992). Essas taxas são superiores às observadas nos muares do presente trabalho. Isso ocorreu porque, nos estudos citados, foi considerada a ocorrência de DA em outras articulações dos equinos estudados. $\mathrm{O}$ fato de terem sido consideradas apenas as DA do carpo nos muares indica que a incidência de DA na população avaliada no presente estudo pode estar subestimada.

A incidência de DA observada nos muares $(37,1 \%)$ deste estudo foi próxima à observada por RIECK et al. (2000), em potros de 30 dias de idade da raça Puro Sangue Inglês (31,6\%). Entretanto, a comparação entre os estudos deve ser feita com cautela, uma vez que a diferença de idade entre os animais de cada trabalho pressupõe diferenças na origem da DA. RIECK et al. (2000) atribuíram origem congênita à ocorrência de DA, enquanto a observada nos muares deste trabalho teve origem adquirida.

Em relação ao tipo de DA, o carpus valgus é a mais comumente observada no carpo dos equinos 
Tabela 1 - Número absoluto de muares e de membros acometidos por DA no carpo por grupo de tratamento distribuídos em cada geração. Os valores porcentuais do tipo de DA são referentes ao número total de membros acometidos em cada grupo.

\begin{tabular}{|c|c|c|c|c|c|c|c|c|c|c|c|c|}
\hline \multirow{2}{*}{ Grupo } & \multicolumn{3}{|c|}{--------No de animais--------- } & \multicolumn{3}{|c|}{-------No de membros------- } & \multicolumn{3}{|c|}{------\% de valgus------ } & \multicolumn{3}{|c|}{------\% de varus------- } \\
\hline & G1 & G2 & Total & G1 & $\mathrm{G} 2$ & Total & G1 & $\mathrm{G} 2$ & Total & G1 & $\mathrm{G} 2$ & Total \\
\hline $\mathrm{TC}$ & 2 & 7 & 9 & 3 & 12 & 15 & 20 & 80 & 100 & 0 & 0 & 0 \\
\hline TEP & 1 & 4 & 5 & 1 & 6 & 7 & 14,3 & 57,1 & 71,4 & 0 & 28,6 & 28,6 \\
\hline GTF & 0 & 2 & 2 & 0 & 4 & 4 & 0 & 0 & 0 & 100 & 0 & 100 \\
\hline Total & 3 & 13 & $16^{*}$ & 4 & 22 & 26 & - & - & - & - & - & - \\
\hline
\end{tabular}

TC: Tratamento conservativo; TEP: Transecção e elevação de periósteo; GTF: TEP e grampo trans-fisário. *O total de animais acometidos por DA no carpo foi maior do que o descrito no texto (13 animais), porque, em três muares, foi instituído um tipo de tratamento para cada membro. Em um animal da G1 e em dois da G2, todos portadores de carpus valgus, um membro foi alocado no grupo TC e o outro no grupo TEP.

(RIECK et al., 2000). Em estudos realizados através de casuística hospitalar, foi observado que a incidência de carpus valgus é de duas a seis vezes maior que a incidência de carpus varus (BERTONE et al., 1985; PHARR \& FRETZ, 1981). Nos muares avaliados, a incidência de carpus valgus foi 2,9 vezes maior do que a de carpus varus. Portanto, a proporção entre as DA que ocorreram no carpo dos muares está de acordo com os valores descritos em equinos.

A incidência de DA foi maior nos muares da G1 quando comparada com G2, o que pode ser explicado pela repetição do protocolo de treinamento e maior tempo de pastejo em Brachiaria humidicola, com o potencial de levar à deficiência de cálcio nestes animais. Entretanto, efeitos relativos ao pastejo de Brachiaria humidicola sobre a incidência de doenças ortopédicas do desenvolvimento ainda não foram descrito em muares.

A necessidade de alguma modalidade de tratamento em $37,1 \%$ dos muares se assemelha com a descrita para equinos (ALDRED, 1998; O’DONOHUE et al., 1992). Porém, a demanda por tratamento cirúrgico dos muares acometidos $(53,8 \%)$ foi 6,7 vezes maior do que a observada nos equinos referidos por ALDRED (1998).

A avaliação da resposta ao tratamento foi feita através da variação da angulação da DA de cada membro. A variação da angulação da DA em funçãodos tratamentos instituídos está ilustrada na figura 1 e os resultados nos animais podem ser observados na figura 2 .

Apesar de READ et al. (2002) relatarem cura espontânea em equinos com DA menor que $8^{\circ}$ e inferior a $13^{\circ}$ associada ao casqueamento, respectivamente,

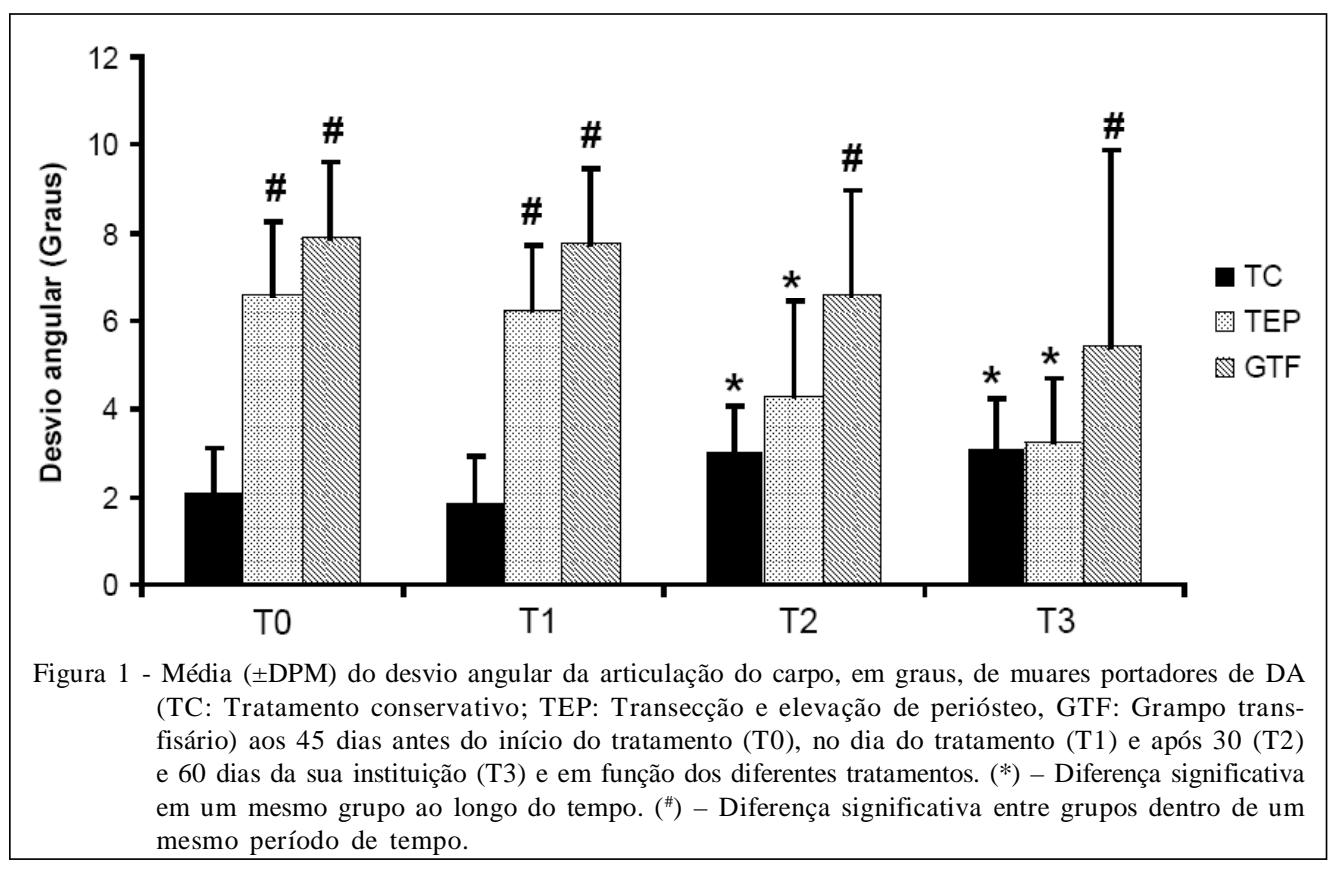

Ciência Rural, v.42, n.10, out, 2012. 


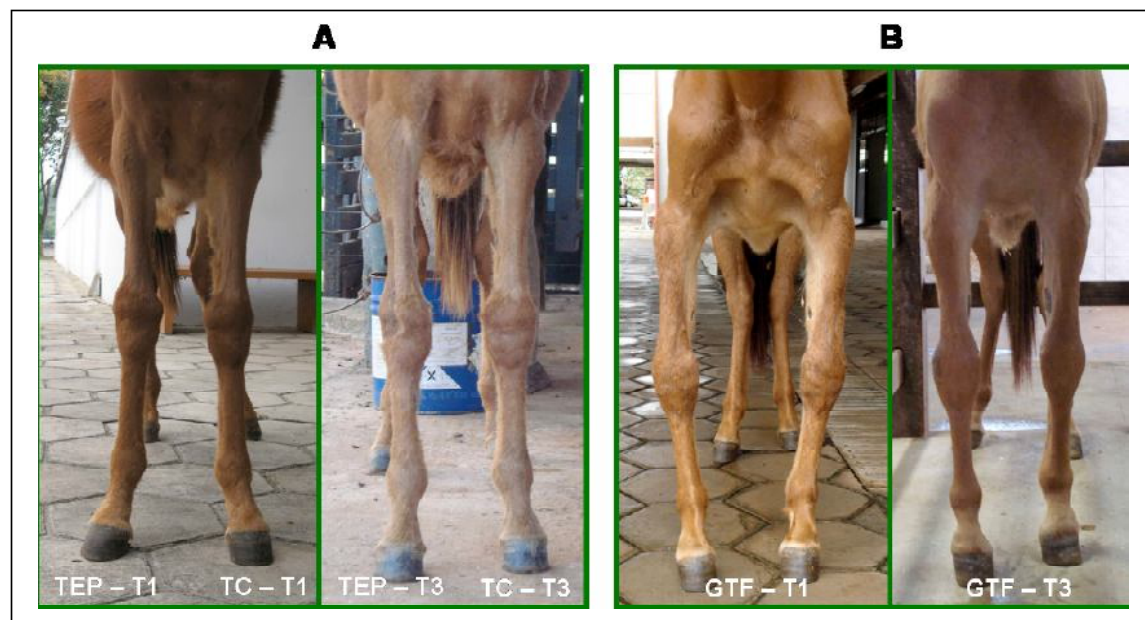

Figura 2 - Vista anterior dos membros torácicos de muares portadores de DA (TC: Tratamento conservativo; TEP: Transecção e elevação de periósteo; GTF: Grampo transfisário) antes da instituição dos tratamentos (T1) e após 60 dias da sua realização (T3). A) Animal pertencente à geração 2, acometido por carpus valgus em ambos os membros. O membro direito foi alocado no grupo TEP e o esquerdo no grupo TC. B) Animal pertencente à geração 1, portador de carpus varus nos dois membros, sendo ambos alocados no grupo GTF.

não foi observada redução significativa da DA nos muares do presente estudo durante os 45 dias que antecederam a instituição dos tratamentos. Isso ocorreu porque os equinos que apresentaram resultados frente ao tratamento conservativo eram mais jovens do que os muares estudados e eram alocados em baias ao invés de piquetes (READ et al., 2002).

$\mathrm{O}$ aumento da angulação observado no grupo TC, a partir de T2, é decorrente do aumento do desvio dos membros dos três animais que tiveram o membro contralateral alocado no grupo TEP. AUER \& MARTENS (1982) relataram aumento gradativo de $1,7^{\circ}$ na angulação dos membros não operados após 60 dias da cirurgia no membro contralateral. Tal aumento foi atribuído ao sobrepeso sobre o membro não operado e ao fato de os muares terem sido mantidos em piquete no período pós-operatório (AUER \& MARTENS, 1982).

Potros submetidos a TEP no carpo com 45 dias de vida apresentaram redução da DA de $3,1^{\circ}$ após três meses (AUER \& MARTENS, 1982). A redução após dois meses, observada nos muares, foi de $3^{\circ}$. Apesar da angulação inicial e final também serem semelhantes, observou-se que, mesmo os muares sendo de idade superior (7,9 meses) à correção da DA, ocorreu em menor período de tempo. WITT \& HUNT (2009) citaram que, em equinos de idade maior, é esperado que a correção do desvio seja menor e mais lenta, devido à menor taxa de crescimento e comentaram ainda que a opção pela TEP, após os oito meses de idade, pode acarretar em insucesso. Portanto, é provável que os muares apresentem resposta a TEP diferente dos equinos, o que permitiria a utilização dessa técnica para a correção de DA em muares com idade avançada.

A redução de $2,3^{\circ}$ da DA, decorrente da associação da TEP à colocação do grampo trans-fisário, observada nos animais do grupo GTF, não foi significativa. Em equinos, foi observado que o sucesso na redução da DA através da utilização de parafusos trans-fisários tem maior chance de ocorrer quando estes são aplicados até os 12 meses de idade (WITTE \& HUNT, 2009). A idade elevada dos muares desse grupo (20 meses) está diretamente relacionada à menor taxa de crescimento e, consequentemente, à menor resposta ao tratamento das DA observados nesse grupo. Apesar da associação de técnicas de estimulação e inibição de crescimento ser recomendada (AUER \& VON RECHENBERG, 2006), nãoépossível avaliar separadamente a influência de cada técnica na redução do desvio.

A remoção do implante é recomendada quando a correção da DA for atingida (BERTONE, 2002; AUER \& VON RECHENBERG, 2006; WITTE \& HUNT, 2009). Entre os membros do grupo GTF, apenas um deles atingiu a angulação de $3^{\circ}$ após dois meses da realização da cirurgia. Devido à falta de expectativa na correção da DA nos demais membros, problemas de logística e financeiros, optou-se pela retirada de todos os grampos simultaneamente. 


\section{CONCLUSÃO}

A implementação de protocolos de treinamento em muares com idade média de quatro meses, junto a uma provável deficiência de cálcio, podem desencadear carpus varus e valgus que demandam tratamento cirúrgico.

A associação da TEP ao casqueamento promove redução do desvio angular do carpo nos membros com angulação $>3^{\circ} \mathrm{e} \leq 7^{\circ}$ em muares com média de idade de 7,9 meses. A realização da TEP em apenas um membro do animal promove aumento do desvio na articulação contralateral. Muares com média de idade de 20 meses com desvio angular no carpo $>7^{\circ}$ bilateral não apresentam melhora significativa ao serem tratados com grampos trans-fisários associado à TEP e ao casqueamento.

\section{COMITÊ DE ÉTICA E BIOSSEGURANÇA}

O presente estudo foi aprovado pelo Comitê de Ética em Experimentação Animal da Universidade Federal de Minas Gerais sob o protocolo número 132/10.

\section{REFERÊNCIAS}

ALDRED, J. Developmental orthopaedic disease in horses. Rural Industries Research and Development Corporation Publications, v. 97/79, p.1-31, 1998. Disponível em: <https:// rirdc.infoservices.com.au/items/97-079>. Acesso em: 19 jun. 2012.

AUER, J.A.; MARTENS, R.J. Periosteal transection and periosteal stripping for correction of angular limb deformities in foals. American Journal Veterinary Research, v.43, p.1530-1534, 1982 .

AUER, J.A.; von RECHENBERG, B. Treatment of angular limb deformities in foals. Clinical Technique Equine Practice, v.5, p.270-281, 2006. Disponível em: <http:// www.sciencedirect.com/science/article/pi i/ S153475160600093X>. Acesso em: 19 jun. 2012. doi: 10.1053/j.ctep.2006.09.001.

BERTONE, A.L. Part V: the carpus. In: STASHAK, T.S. Adam's lameness in horses. 5.ed. Baltimore: Williams \& Wilkins, 2002. p.830-863.

BERTONE, A.L. et al. Periosteal transaction and stripping for treatment of angular limb deformities in foals: radiographic observations. Journal American Veterinary Medical Association, v.187, p.153-156, 1985.

CASTON, S.S. et al. How to make, place, and remove transphyseal staples. In: ANNUAL CONVENTION OF THE AMERICAN ASSOCIATION OF EQUINE PRACTITIONERS.
- AAEP, Proceedings... Orlando, Florida, USA. American Association of Equine Practitioners. v. 53, 2007. Disponível em: <http://www.ivis.org/proceedings/aaep/2007/caston/ chapter.asp?LA=1>. Acesso em: 19 jun. 2012.

GREET T.R.C.; CURTIS, S.J. Foot management in the foal and wealing. Veterinary Clinics of North America: Equine Practice, v.19, p.501-517, 2003. Disponível em: <http:// www.sciencedirect.com/science/article/pii/S0749073903000166>. Acesso em: 19 jun. 2012. doi: 10.1016/S0749-0739(03)00016-6.

LEPEULE, J. et al. Association of growth, feeding practices, and exercise conditions with the prevalence of developmental orthopaedic disease in limbs of french foals at weaning. Preventive Veterinary Medicine, v.89, p.167-177, 2009. Disponível em: <http://www.sciencedirect.com/science/article/ pii/S0167587709000439>. Acesso em: 19 jun. 2012. doi: 10.1016/j.prevetmed.2009.02.018.

McILWRAITH, C.W. Developmental orthopedic disease: problems of limbs in young horses. Journal Equine Veterinary Science, v.24, p.475-479, 2004. Disponível em: <http://www.sciencedirect.com/science/article/pii/ S0737080604004009>. Acesso em: 19 jun. 2012. doi: 10.1016/ j.jevs.2004.10.004.

O'DONOHUE, D.D. et al. The incidence of abnormal limb development in the Irish Thoroughbred from birth to 18 months. Equine Veterinary Journal, v.24, p.305-309, 1992.

PHARR, J.W.; FRETZ, P.B. Radiographic findings in foals with angular limb deformities. Journal American Veterinary Medical Association, v.179, p.812-817, 1981.

PUOLI FILHO, J.N.P. et al. Suplementação mineral e mobilização de cálcio nos ossos de equinos em pastagem de Brachiaria humidicola. Pesquisa Agropecuaria Brasilera, v.34, p.873-878, 1999. Disponível em: <http://www.scielo.br/ pdf/pab/v34n5/8435.pdf>. Acesso em: 19 jun. 2012.

READ, E.K. et al. Effect of hemi-circumferential periosteal transaction and elevation in foals with experimentally induced angular limb deformities. Journal American Veterinary Medical Association, v.221, p.536-540, 2002. Disponível em: <http://avmajournals.avma.org/doi/pdf/10.2460/ javma.2002.221.536>. Acesso em: 19 jun. 2012. doi: 10.2460/ javma.2002.221.536.

RIECK, S.E. et al. Desvios angulares em potros puro sangue de corrida do nascimento aos 30 dias de vida: origem e incidência. Ciência Rural, v.30, p.825-828, 2000. Disponível em: <http:/ /www.scielo.br/pdf/cr/v30n5/a14v30n5.pdf>. Acesso em: 19 jun. 2012.

WITTE, S.; HUNT, R. A review of angular limb deformities. Equine Veterinary Education, v.21, p.378-387, 2009. Disponível em: <http://onlinelibrary.wiley.com/doi/10.2746/ 095777309X440096/abstract>. Acesso em: 19 jun. 2012. doi: $10.2746 / 095777309 \times 440096$. 